\title{
NA62 RICH performance: measurement and optimization
}

\author{
G. Anzivino ${ }^{\mathrm{a}, \mathrm{b}}$, M. Barbanera ${ }^{\mathrm{b}}$, A. Bizzeti ${ }^{\mathrm{c}, \mathrm{d}}$, F. Brizioli $^{\mathrm{a}, \mathrm{b}}, \mathrm{F}$. Bucci $^{\mathrm{d}}$, A. Cassese $^{\mathrm{d}, \mathrm{e}}$, P. Cenci $^{\mathrm{b}}$, \\ B. Checcucci ${ }^{b}$, R. Ciaranfi ${ }^{d}$, V. Duk ${ }^{\text {f,* }}$, J. Engelfried ${ }^{g}$, N. Estrada-Tristan ${ }^{\text {g,h }}$, E. Iacopini ${ }^{\text {d,e }}$, \\ E. Imbergamo ${ }^{\mathrm{a}, \mathrm{b}}$, G. Latino ${ }^{\mathrm{d}, \mathrm{e}}$, M. Lenti $^{\mathrm{d}, \mathrm{e}}$, R. Lollini $^{\mathrm{a}, \mathrm{b}}$, M. Pepe ${ }^{\mathrm{b}}$, M. Piccini $^{\mathrm{b}}$, R. Volpe ${ }^{\mathrm{d}, \mathrm{e}}$ \\ ${ }^{\text {a }}$ Dipartimento di Fisica e Geologia dell'Università di Perugia, Italy \\ ${ }^{\mathrm{b}}$ INFN - Sezione di Perugia, Italy \\ ${ }^{\mathrm{c}}$ Dipartimento di Scienze Fisiche, Informatiche e Matematiche dell'Università di Modena e Reggio Emilia, Italy \\ ${ }^{\mathrm{d}}$ INFN - Sezione di Firenze, Italy \\ ${ }^{\mathrm{e}}$ Dipartimento di Fisica e Astronomia dell'Università di Firenze, Italy \\ ${ }^{\mathrm{f} S c h o o l}$ of Physics and Astronomy, University of Birmingham, United Kingdom ${ }^{1}$ \\ ${ }^{\mathrm{g}}$ Instituto de Física, Universidad Autónoma de San Luis Potosí, Mexico ${ }^{2}$ \\ ${ }^{\mathrm{h}}$ Departamento de Ciencias e Ingeniería, Universidad de Guanajuato, Guanajuato, Mexico
}

\section{A R T I C L E I N F O}

\section{Keywords:}

Kaon decays

RICH detector

Detector performance

\begin{abstract}
A B S T R A C T
The Ring Imaging Cherenkov detector of the NA62 experiment at CERN SPS was commissioned in 2014, but the optimal performance was achieved in 2016 after the precise mirror alignment with reconstructed tracks. The measurement and monitoring of basic performance parameters is discussed: ring radius resolution, ring centre resolution, single hit resolution and mean number of hits per ring. The performance is measured with 2016 data on the positron sample. Different contributions to the resolutions are calculated.
\end{abstract}

\section{Introduction}

The main goal of the NA62 experiment running at CERN is the $10 \%$ precision measurement of the branching ratio of a rare decay $K^{+} \rightarrow \pi^{+} v \bar{v}$. Fig. 1 represents the experimental setup described in detail in [1].

One of crucial detectors of the NA62 setup is the Ring Imaging CHerenkov detector (RICH) detector. It identifies charged particles from kaon and pion decays and provides the reference time for the L 0 trigger. The design and commissioning of the RICH detector are summarized in [2]. The detector layout is shown in Fig. 2.

The RICH mirror system [2] consists of 18 hexagonal (350 mm side) and two semi-hexagonal mirrors, the latters placed in the central part. The focal length of all mirrors is $f=17 \mathrm{~m}$. Mirror orientation is provided by two stabilizing aluminium ribbons connected to the mirror at one end and to a piezo motor at the other end. A third anti-rotating ribbon prevents the mirror rotation around the longitudinal axis.

All mirrors are grouped into two parts referred to as Jura and Saleve, each group having the same centre of curvature to the left and to the right of the beam pipe. Such division allows to avoid light loss due to interactions with the beam pipe.

In the focal plane of each group there is a photomultiplier (PM) disk containing 976 PMs. Winston cones [3] are used to enhance light collection. The cone outer diameter $\mathrm{d}_{\text {cone }}=18 \mathrm{~mm}$ coincides with that of PM, the inner diameter is equal to the diameter of the PM sensitive area $\mathrm{d}_{P M}=7.5 \mathrm{~mm}$.

\section{Performance measurement}

The quantitative parameters of the RICH particle identification are determined by more fundamental performance parameters like single hit resolution and the average number of hits per event. These parameters are usually evaluated for electron/positron tracks.

In the following Sections the measurement of the basic performance is described: ring radius resolution, ring centre resolution, single hit resolution and mean number of hits per ring. The 2016 data sample is used for the analysis. Events with one positron ring fully contained in the RICH acceptance are selected, using the information from the electromagnetic calorimeter and spectrometer (for the electron identification).

\section{Precise mirror alignment}

To provide the best detector performance, RICH mirrors must be aligned as precisely as possible. The accuracy of the laser alignment performed during the installation is $\sim 500 \mu \mathrm{rad}$ in terms of mirror

\footnotetext{
* Corresponding author.

E-mail address: Viacheslav.Duk@cern.ch (V. Duk).

1 Funded by the EU Horizon 2020 research and innovation programme (Marie Sklodowska-Curie grant No 701386).

2 Funded by Consejo Nacional de Ciencia y Tecnología (CONACyT) and Fondo de Apoyo a la Investigación (UASLP).
} 


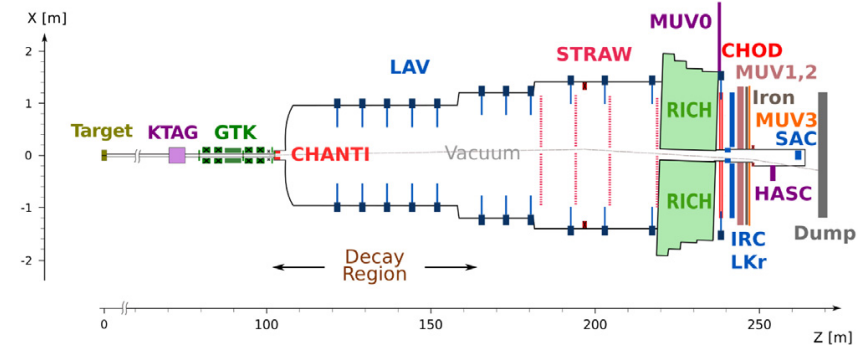

Fig. 1. NA62 experimental setup. The beam goes in the positive $\mathrm{Z}$ direction. The positive direction of the $\mathrm{Y}$ axis is vertical.

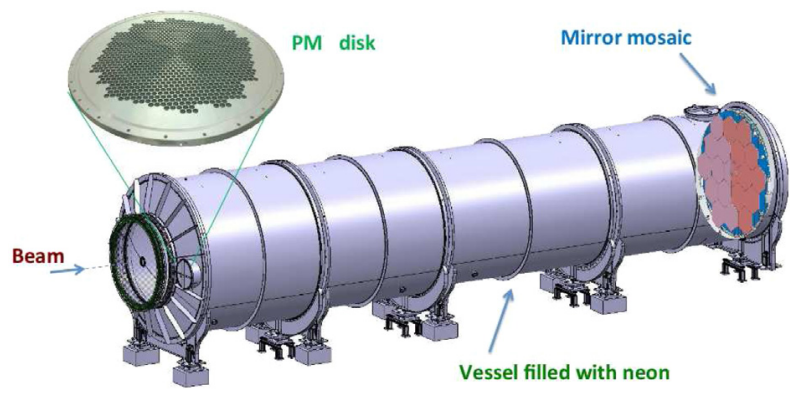

Fig. 2. RICH detector. One of two photomultiplier disks is zoomed. The mirror group shown in dark pink reflects light towards the zoomed disk, while the other half shown in light pink is oriented towards the second disk.

orientation [2]. A better precision is achieved by using reconstructed tracks. The precise mirror alignment is described in detail in [4]. Mirrors of each group (Jura, Saleve) are aligned with respect to a reference mirror. First, the absolute misalignment with respect to the nominal orientation is measured for each mirror. Second, the relative misalignment with respect to the reference mirror is calculated and translated to the piezo motor movement needed to compensate the relative misalignment. Third, piezo motors are moved according to the calculations and the absolute misalignment is measured again. The procedure is repeated until the final accuracy is achieved: $\sim 30 \mu \mathrm{rad}$ in terms of the mirror orientation, or $\sim 1 \mathrm{~mm}$ in terms of the ring centre position. Finally, global offsets (a global offset is the average absolute misalignment of a group) and the residual misalignment (misalignment of a mirror with respect to a corresponding global offset) are calculated. The results are shown in Fig. 3. The alignment precision is limited by hysteresis effects in the ribbon-mirror system: for small movements the piezo motor movement is no longer proportional to the ring centre displacement.

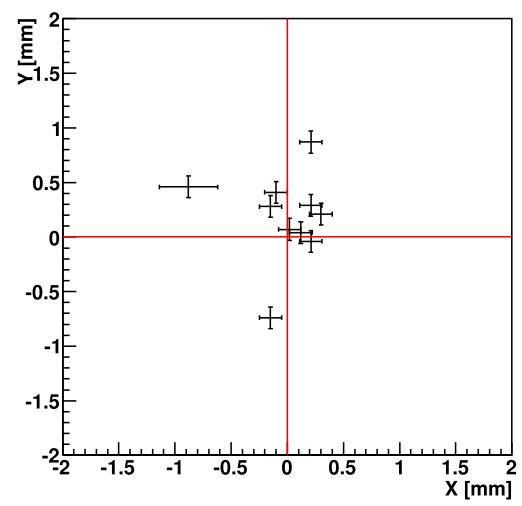

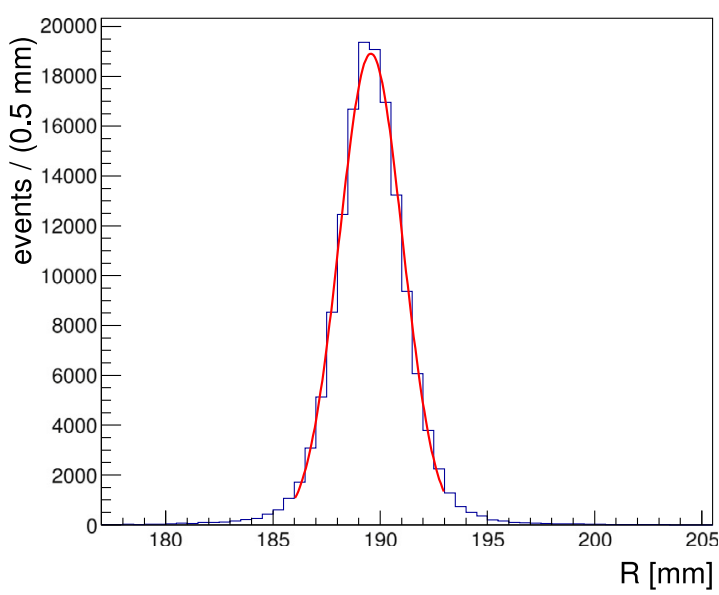

Fig. 4. Positron ring radius. A gaussian fit is performed: $\langle R\rangle=189.6 \mathrm{~mm}, \sigma_{R}=1.47 \mathrm{~mm}$. The figure is taken from [4]. (C) SISSA Medialab Srl. Reproduced by permission of IOP Publishing. All rights reserved.

The misalignment measurement is performed on a monthly basis during the data taking. Global offsets and residual misalignment are stored in a database.

\section{Ring radius resolution}

Fig. 4 demonstrates the ring radius distribution. The mean value $\langle R\rangle=189.6 \mathrm{~mm}$ corresponds to the Cherenkov angle of $11.2 \mathrm{mrad}$. The gaussian width of the distribution gives the ring radius resolution: $\sigma_{R}=$ $1.47 \mathrm{~mm}$, or $90 \mu \mathrm{rad}$.

The mean value is used to calculate the neon refractive index and is monitored on a daily basis. The values are stored in a database and used at the analysis level.

\section{Ring centre resolution}

Fig. 5 shows the difference between the measured and expected ring centre position (in $\mathrm{X}$ and $\mathrm{Y}$ ) which is fitted by the gaussian. The expected position is calculated using the trajectory measured by the spectrometer. The ring centre resolution is given by the gaussian width of this distribution, the contribution from the spectrometer being negligible. The obtained values (2.96 and $2.92 \mathrm{~mm}$ ) correspond to 170 $\mu \mathrm{rad}$ in terms of the track slope resolution.

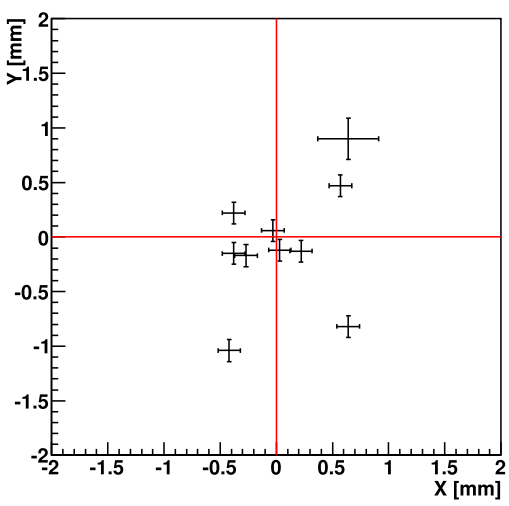

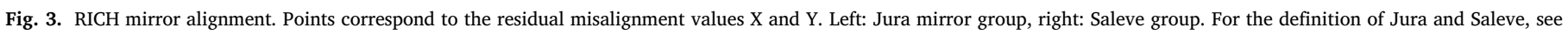
Section 1. Each point corresponds to one mirror. The figure is taken from [4]. ( ) SISSA Medialab Srl. Reproduced by permission of IOP Publishing. All rights reserved. 

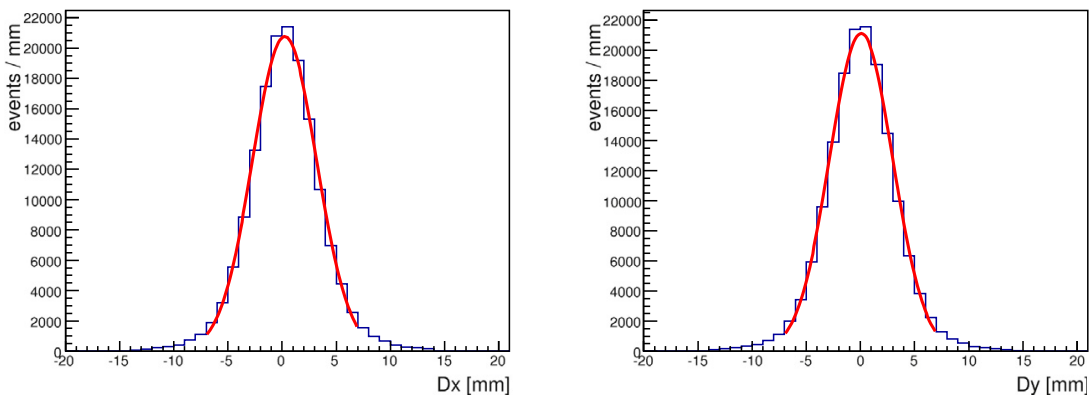

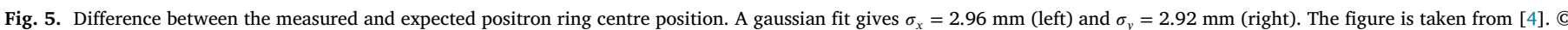
SISSA Medialab Srl. Reproduced by permission of IOP Publishing. All rights reserved.

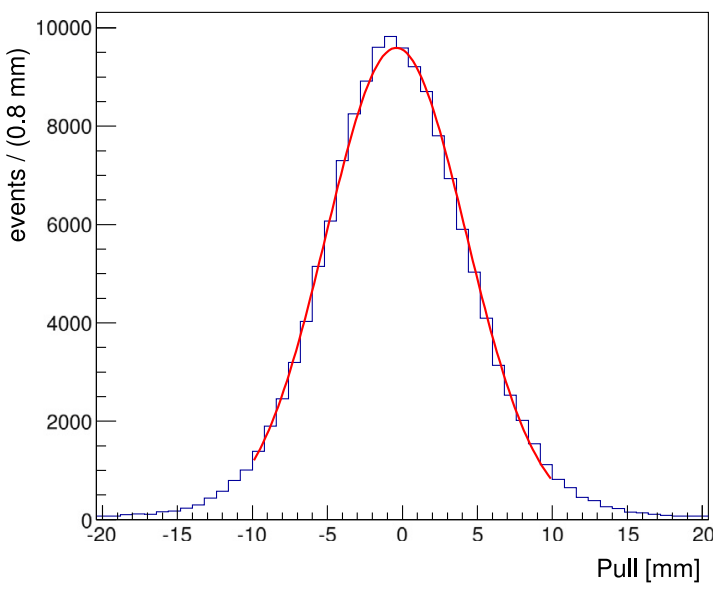

Fig. 6. Pull distribution. A gaussian fit is performed: $\sigma_{\text {hit }}=4.66 \mathrm{~mm}$. The figure is taken from [4]. (C) SISSA Medialab Srl. Reproduced by permission of IOP Publishing. All rights reserved.

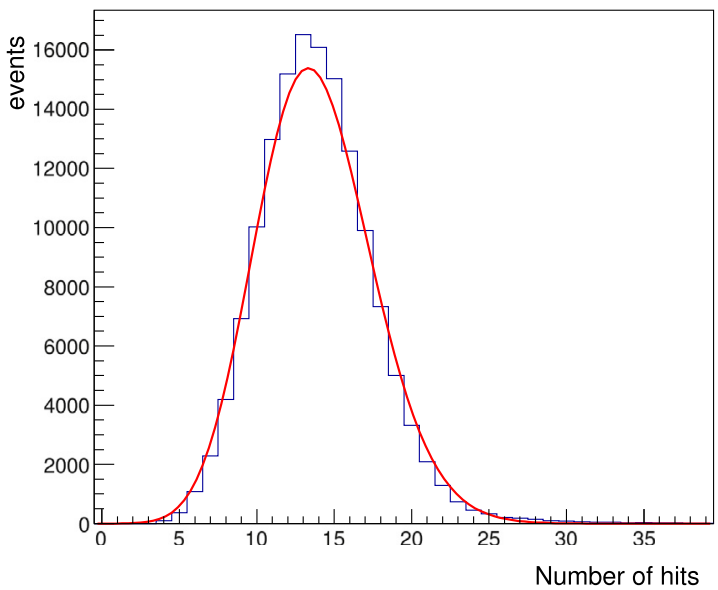

Fig. 7. Number of hits per ring distribution. A Poissonian fit is performed: $\left\langle N_{\text {hits }}\right\rangle=$ 13.8. The figure is taken from [4]. (C) SISSA Medialab Srl. Reproduced by permission of IOP Publishing. All rights reserved.

\section{Single hit resolution}

To calculate the single hit resolution, a Pull variable is introduced: Pull $=\left(\mathrm{R}-\mathrm{R}_{\text {exp }}\right) \sqrt{N_{\text {hits }}-3}$. Here $\mathrm{R}$ is the measured ring radius, $\mathrm{R}_{\text {exp }}$ is the radius calculated from the measured momentum for the positron mass, $\left(\mathrm{N}_{\text {hits }}-3\right)$ is the number of degrees of freedom of the ring fit. The single hit resolution $\sigma_{h i t}$ is obtained from the gaussian width of the Pull distribution shown in Fig. 6; $\sigma_{\text {hit }}=4.66 \mathrm{~mm}(270 \mu \mathrm{rad})$.
Table 1

Toy MC and data comparison. $M C$ is the quadratic sum of the multiple scattering and the geometry contributions. Single mirror stands for the data sample with rings in a single mirror.

\begin{tabular}{lllll}
\hline Parameter & Multiple scattering & Geometry & MC & Single mirror \\
\hline$\sigma_{R}(\mathrm{~mm})$ & 0.4 & 1.2 & 1.3 & 1.3 \\
$\sigma_{\text {hit }}(\mathrm{mm})$ & 1.4 & 3.9 & 4.1 & 4.2 \\
$\sigma_{x}(\mathrm{~mm})$ & 1.4 & 2.2 & 2.6 & 2.8 \\
\hline
\end{tabular}

Table 2

Resolution summary. Misalignment stands for the mirror misalignment contributions. Measured corresponds to the values measured with the data.

\begin{tabular}{lllll}
\hline Parameter & Misalignment & Multiple scattering & Geometry & Measured \\
\hline$\sigma_{R}(\mathrm{~mm})$ & 0.7 & 0.4 & 1.2 & 1.5 \\
$\sigma_{\text {hit }}(\mathrm{mm})$ & 2.1 & 1.4 & 3.9 & 4.7 \\
$\sigma_{x}(\mathrm{~mm})$ & 0.9 & 1.4 & 2.2 & 3.0 \\
\hline
\end{tabular}

The contribution to $\sigma_{h i t}$ due to the neon dispersion [5] is found to be small. The detailed calculation (see [4] for details) gives $\sigma_{\text {hit, } \Delta n} \simeq 0.6 \mathrm{~mm}$.

The contribution to $\sigma_{\text {hit }}$ due to the mirror misalignment is measured directly by comparing the event sample with all rings and the subsample with rings contained in a single mirror: $\sigma_{\text {hit, mirror }}=2.1 \mathrm{~mm}$.

Another important contribution comes from the multiple scattering at the entrance window ( $2 \mathrm{~mm}$ of aluminium, $\sim 2.2 \% X_{0}$ ) and in neon $\left(18 \mathrm{~m}, \sim 5.6 \% X_{0}\right)$. To calculate the effect, a toy Monte Carlo (MC) has been developed that simulates the smearing of the track direction (due to scattering) and photon emission points in neon. The gaussian width of the obtained Pull distribution gives the value of the scattering contribution: $\sigma_{\text {hit, scattering }}=1.39 \mathrm{~mm}$.

The geometry contribution dominates the single hit resolution, it is determined by the outer and inner Winston cone diameter. If all the incident light was collected by the cone, the geometry contribution would be equal to $\sigma_{\text {geom, } \max }=d_{\text {cone }} / 4=4.5 \mathrm{~mm}$. In the case of absorbing cone surface, the geometry contribution would be given by the diameter of the sensitive region of PMs: $\sigma_{\text {geom, } \min }=d_{P M} / 4=1.9 \mathrm{~mm}$. To estimate realistic geometry contribution, a toy MC has been developed. Ring centres are uniformly generated in two-dimensional grid of Winston cones. Photons impact points are distributed on the ring, $N_{\text {photons }}$ is given by the Poissonian p.d.f. with the known average value. Hits are assigned to the closest cone centre coordinate and $5 \%$ of hits are rejected if the impact points are within the mylar area (to take into account the cone reflectivity). The gaussian width of the Pull distribution for survived hits gives an estimate of the geometry contribution: $\sigma_{\text {hit, geom }}=3.9 \mathrm{~mm}$.

To validate the toy MC, multiple scattering and cone geometry effects are combined and the resolutions are compared to the ones obtained from the data with rings contained in a single mirror (to exclude the mirror misalignment contribution). The results are summarized in Table 1. Reasonable agreement is observed.

Table 2 summarizes all contributions to resolutions. 


\section{Number of hits per ring and Figure of Merit}

Fig. 7 shows the number of hits distribution. The average value is $\left\langle N_{\text {hits }}\right\rangle=13.8$. During the data taking $\left\langle N_{\text {hits }}\right\rangle$ is measured on a daily basis, the values are stored in a database and can be used at the analysis level.

The Figure of Merit is calculated using the obtained value of $\left\langle N_{\text {hits }}\right\rangle$ : $\mathrm{N}_{0}=\left\langle N_{\text {hits }}\right\rangle /\left(L \sin ^{2} \theta\right)$, here $\mathrm{L}$ is the vessel length and $\theta$ is the Cherenkov angle. The obtained value $\mathrm{N}_{0} \sim 65 \mathrm{~cm}^{-1}$ can be used to evaluate the performance of the whole detector as compared to other RICH detectors.

\section{Conclusion}

The basic performance of the RICH detector has been measured using the positron tracks. The ring radius resolution is $1.5 \mathrm{~mm}$, the ring centre resolution is 3.0 (2.9) $\mathrm{mm}$ for $\mathrm{X}(\mathrm{Y})$ coordinate, the single hit resolution is $4.7 \mathrm{~mm}$, the average number of hits per ring is 13.8 . The performance is optimized by the precise mirror alignment, the accuracy being $\sim 30$ $\mu \mathrm{rad}$ in terms of the mirror angular orientation. The main performance parameters are periodically monitored and the values are stored in a database.

\section{References}

[1] E. Cortina Gil, et al., JINST 12 (2017) P05025.

[2] D. Aisa, et al., JINST 12 (2017) P12017.

[3] H. Hinterberger, R. Winston, Rev. Sci. Instrum. 37 (1966) 1094.

[4] G. Anzivino, et al., JINST 13 (2018) P07012.

[5] A. Bideau-Mehu, et al., J. Quant. Spectrosc. Radiat. Transfer 25 (1981) 395. 